\title{
Praktische Dosimetrie und Konstanzprüfung zur Einführung der intraoperativen Bestrahlung mit Intrabeam ${ }^{\circledR}$ (Zeiss)
}

Practical dosimetry and constancy check at introduction of intraoperative radiotherapy with Intrabeam ${ }^{\circledR}$ (Zeiss)

Petra Maria Härtl†, Barbara Dobler, Oliver Kölbl, Marius Treutwein

Universitätsklinikum Regensburg, Klinik und Poliklinik für Strahlentherapie

Received 2 March 2009, Accepted 9 September 2009, Available online 16 October 2009

doi:10.1016/j.zemedi.2009.09.001

\section{Zusammenfassung}

Vorgestellt wird eine Methode zur Überprüfung der Dosimetrie am intraoperativen Bestrahlungssystem Intrabeam ${ }^{\circledR}$ (Zeiss) mit 50-kV-Röntgenstrahlung. Außerdem wurde die Langzeitstabilität des Gerätes in Bezug auf Dosis und deren Isotropie mit dem vom Hersteller vorgegeben Verfahren überprüft.

Für die Absolutdosimetrie wurde ein Elektrometer des Typs Unidos ${ }^{\circledR}$ mit einer für Wasserenergiedosis kalibrierten Weichstrahlkammer (TM 23342) der Firma PTW, Freiburg verwendet. Als Phantom diente das Plattenphantom PTW Typ 2962. RW1-Platten wurden als Aufbaumaterial benutzt. Um den seitlichen Streubeitrag zu berücksichtigen, wurden die Applikatoren in einen Beutel mit Wasser eingebracht.

Unsere Untersuchungen ergaben folgende Ergebnisse: Die Dosis an der Oberfläche wich im Mittel um 3\% von der geräteinternen Dosimetrie ab. Die Konstanzprüfung der Dosisleistung am Referenzort der Messvorrichtung ergab eine mittlere Abweichung von 0,3\%. Die Messung der Dosisverteilung senkrecht zur Geräteachse $\mathrm{z}$ (Referenz)ergab folgende mittlere Abweichungen: $\mathrm{x}-2,7 \%, \mathrm{y}-4,1 \%,-\mathrm{x}-7,1 \%$ und für $-\mathrm{y}-5,3 \%$.

Die vorgestellte Methode zur Überprüfung der Absolutdosimetrie führt zu gut reproduzierbaren Ergebnissen. Diese stimmen innerhalb der Toleranzen $\pm 10 \%$ mit der Dosimetrie der Fa. Zeiss überein. Da die Konstanz der Dosisleistung arbeitstäglich geprüft 
wird, ist es ausreichend, die Absolutdosimetrie vor Inbetriebnahme beziehungsweise beim Tausch der Applikatoren durchzuführen. Zur Konstanzprüfung sind die vom Hersteller vorgegebenen Prüfungen geeignet.

Die Ergebnisse der Konstanzprüfung zeigen eine hohe Langzeitstabilität in Bezug auf Dosisleistung und Symmetrie.

\section{Abstract}

The check of dosimetry of the intraoperative radiotherapy system Intrabeam ${ }^{\circledR}$ is predefined by the manufacture (Zeiss). The purpose of the study was to develop and implement a method to verify the internal dosimetry of Intrabeam ${ }^{\circledR}$ (Zeiss). Additionally the long-term stability of Intrabeam ${ }^{\circledR}$ was checked for dose and isotropy.

For dose to water measurements an Unidos ${ }^{\circledR}$ was combined with a soft jet chamber (TM 23342) which was calibrated in water absorbed dose and as a phantom the type 2962 (PTW Freiburg) was used. RW1 plates were inserted as build up material. The applicators were placed in a bag filled with water to consider the side-scattering.

At the surface of the applicator there was a mean difference of 3 percent between the dose to water measurement and the internal dosimetry. The constancy of the dose rate showed a mean deviation of $0.3 \%$ at the reference point. The analysis of the dose distribution perpendicular to the applicator axis $\mathrm{z}$ (reference $\mathrm{z}$-axis) resulted in a mean deviation of $-2.7 \%$ (x-direction) and $-7,1 \%(-\mathrm{x}$-direction) for the $\mathrm{x}$-axis and, respectively $-4.1 \%$ (y-direction) and $-5.3 \%(-\mathrm{y}-$ direction) for the $y$-axis.

The proposed method is suitable to verify the absolute dose of Intrabeam ${ }^{\circledR}$. The dose values measured by this method were congruent to the dosimetry of the manufacture (Zeiss). From our point of view it is sufficient to verify the absolute dosimetry only at time of commissioning of the system or in the case of changing the applicator. For the daily routine the check of constancy specified by the manufacture is adequate, because the dose rate is checked on a daily basis. Additionally the test of constancy showed a high long-term stability in terms of dose rate and symmetry.

\section{Schlüsselwörter}

Brustkrebs; intraoperative Strahlentherapie (IORT); Intrabeam

\section{Keywords}

Breast cancer; intraoperative Radiotherapy (IORT); Intrabeam

\section{Einleitung}

Die intraoperative Strahlentherapie bei Mammakarzinom mit Intrabeam ${ }^{\circledR}$ (Zeiss), sei es im Rahmen der Targetstudie oder als vorgezogener Boost, findet immer mehr Verbreitung [1], [2], [3] and [4]. Es handelt sich hierbei nicht nur um eine Strahlenquelle, sondern um ein komplettes System aus Strahlenquelle, Applikatoren, Dosimeter mit Kammer und Software [5]. Die Dosimetrie bestehend aus Tiefendosiskurven, Dosisleistung und 
Schwächungsfaktoren der Applikatoren und ist werkseitig vorgegeben. Der Anwender kann diese Daten nicht bearbeiten. Das Messverfahren des Herstellers wurde bereits in der Literatur beschrieben [6]. Die Konstanzprüfung der Dosisleistung und der Isotropie erfolgt mit zum System gehörenden Prüfvorrichtungen nach Vorgabe des Herstellers. Zusätzlich sollte eine eigene dosimetrische Überprüfung der Röntgenquelle mit den verwendeten Applikatoren erfolgen [7], [8] and [9]. Da die Verschreibung aus Gründen der Standardisierung auf die Applikatoroberfläche erfolgt [5], [6] and [10] wurde die Dosis an dieser Stelle überprüft. Ergänzend empfiehlt sich eine punktweise Überprüfung der Tiefendosis. Schwierigkeiten bei der Messung bereiten die kugelförmigen Applikatoren mit ihrer schalenförmigen Dosisverteilung. Da es sich bei der verwendeten Strahlenqualität um Weichstrahlung, überwiegende Wechselwirkung in Wasser ist der Photoeffekt, handelt, können die seitlichen Streubeiträge nicht vernachlässigt werden. Messungen im Wasserphantom sind aufwändig, da die Weichstrahlkammer nicht wasserdicht ist. In dieser Arbeit soll eine einfache Methode zur Überprüfung der Wasserenergiedosis vorgestellt werden. In der Regel befindet sich das Bestrahlungssystem nicht in der strahlentherapeutischen Abteilung. Deshalb wurde besonders darauf geachtet, dass nur Hilfsmittel verwendet wurden, die leicht transportierbar sind und ohne großen Aufwand einsetzbar sind. Außerdem werden die Ergebnisse der arbeitstäglich vorgesehenen Konstanzprüfung über den Zeitraum von einem Jahr vorgestellt.

\section{Material und Methoden}

Die Messungen wurden an der intraoperativen Bestrahlungsvorrichtung Intrabeam ${ }^{\circledR}$ (Zeiss) bei einer Energie von $50 \mathrm{kV}$ durchführt. Eine Beschreibung des Aufbaus der Röntgenröhre findet sich bei Armoogum K et al. [11].

\section{Bestimmung der Wasserenergiedosis}

Dosimetrisch untersucht wurden die therapeutisch verwendeten Applikatoren mit einem Durchmesser ab $3 \mathrm{~cm}$. Die mitgelieferten kleineren Applikatoren werden für die Bestrahlung der Brust nicht verwendet, da sie in der Regel die Operationshöhle nicht ausfüllen. Die Bestimmung der Wasserenergiedosis erfolgte mit einem Dosimetriesystem der Fa. PTW. Verwendet wurde ein Unidos ${ }^{\circledR}$ mit einer in Wasserenergiedosis kalibrierten Weichstrahlkammer TM 23342 und das Plattenphantom Typ 2962 mit PMMA als Rückstreumaterial [12]. Um den seitlichen Streubeitrag zu berücksichtigen wurden die Applikatoren in einen Beutel mit Wasser eingebracht (Abb. 1). 


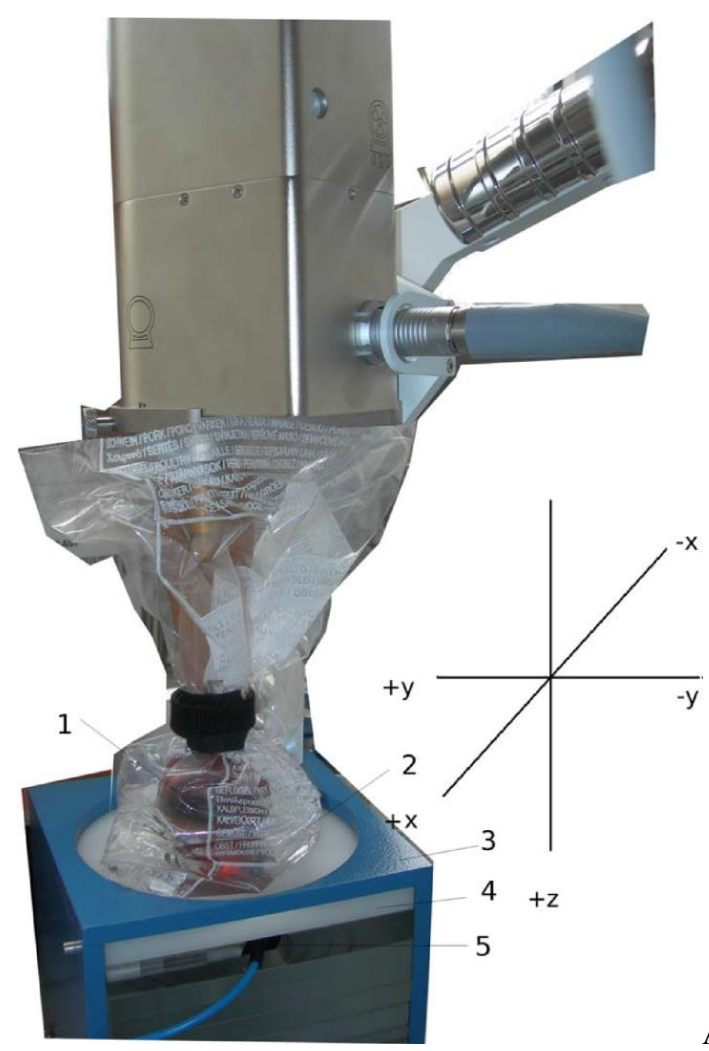

Abbildung 1.

Messaufbau mit Bezugssystem: Die Strahlenquelle mit Applikator (1) befindet sich in einem mit Wasser gefüllten Beutel (2). Der Applikator wird auf das Weichstrahlphantom (3) aufgesetzt. Die Abbildung zeigt den Messaufbau zur Bestimmung der Wasserenergiedosis in $1 \mathrm{~cm}$ Tiefe. Die RW1 Platte (4) wurde für die Dosismessung an der Oberfläche entfernt und der Applikator direkt auf der Weichstrahlkammer (5) positioniert.

Für die Messungen am Referenzort der Dosisverschreibung (Applikatoroberfläche) wurde der Applikator so über der Kammer positioniert, dass sich zwischen ihm und der Kammer nur die Folie des Beutels befand. Zusätzlich zur Dosis an der Applikatoroberfläche wurden die Tiefendosiswerte in $5 \mathrm{~mm}$ und $10 \mathrm{~mm}$ Tiefe überprüft. Als Schwächungsmaterial wurde RW1 verwendet [13] and [14]. Die von uns gemessenen Dosiswerte wurden mit den entsprechenden Dosisangaben des Intrabeamsystems an der Applikatoroberfläche, in 0,5 cm und $1,0 \mathrm{~cm}$ Tiefe verglichen.

Bei allen Messungen wurde die Dosis über eine Messzeit von einer Minute integriert. Von entscheidender Bedeutung ist die Reproduzierbarkeit der Messwerte. Zu diesem Zweck wurden die Applikatoren positioniert, die Dosis gemessen und anschließend der Applikator neu positioniert und die Dosis erneut bestimmt.

\section{Langzeitverhalten der Symmetrie der Röntgenstrahlung und der Dosisleistung}

Der Ablauf der Konstanzprüfung wird durch die Gerätesoftware vorgegeben und erfasst [15]. Ist diese unvollständig oder die Abweichung außerhalb der vom Hersteller vorgesehenen Toleranzen, kann keine Bestrahlung gestartet werden.

Die Überprüfung der Symmetrie-Einstellung erfolgt mit einem zur Ausstattung gehörenden Photodioden-Array (PDA). In diesem befinden sich jeweils eine Diode in $\pm x, \pm y$, und $z$ Richtung (Orientierung Abbildung 1). Die durch die Röntgenstrahlung erzeugte Spannung an der Diode in z-Richtung dient als Referenz. Die erzeugte Spannung an den Dioden $\mathrm{x}, \mathrm{y},-\mathrm{x}$, 
-y wird prozentual bezüglich z ausgeben. Übersteigt die Abweichung 12\%, gilt die Prüfung als nicht bestanden, dann kann keine Behandlung durchgeführt werden.

Die Dosisleistung wird mit einer zur Ausstattung gehörenden Kombination von Weichstrahlkammer TM 23342 mit Unidos überprüft. Die zur Geräteausstattung gehörende Sondenzentriervorrichtung und Ionisationskammerhalterung PAICH (Probe Adjuster Ion Chamber Holder) erlauben unter Berücksichtigung von Temperatur und Luftdruck eine Konstanzprüfung der Dosisleistung an einem festgelegten Messort.

\section{Ergebnisse}

\section{Messung der Wasserenergiedosis}

Die Ergebnisse unserer Messung sind in Tabelle 1 dargestellt. Abhängig von der Größe des Applikators betrug die Dosis in $5 \mathrm{~mm}$ Tiefe, bezogen auf die Applikatoroberfläche, zwischen 47\% und 58\%. In $10 \mathrm{~mm}$ Tiefe lag der Wert zwischen 25\% und 36\%. Eine Berechnung der Halbwertstiefe für verschiedene Applikatoren mit der geräteinternen Software ergab Werte von 4,6 bis $5,2 \mathrm{~mm}$.

Tabelle 1.

Gemessene Dosis pro Minute und deren Abweichung bezogen auf die geräteinterne Dosimetrie an der Oberfläche, in $5 \mathrm{~mm}$ und in $10 \mathrm{~mm}$ Tiefe.

\begin{tabular}{lllllll}
\hline Applikator/Tiefe mm & $\begin{array}{l}\text { Dosis Gy } \\
0\end{array}$ & $\Delta \%$ & $\begin{array}{l}\text { Dosis Gy } \\
5\end{array}$ & $\Delta \%$ & $\begin{array}{l}\text { Dosis Gy } \\
10\end{array}$ & $\Delta \%$ \\
\hline$\phi \mathrm{cm}$ & & & & & & \\
3 & 0,79 & $-3,4 \%$ & 0,41 & $-2,9 \%$ & 0,23 & $-3,3 \%$ \\
3,5 & 1,02 & $-5,3 \%$ & 0,49 & $-4,9 \%$ & 0,26 & $-8,7 \%$ \\
4 & 0,76 & $-1,8 \%$ & 0,35 & $-10,0 \%$ & 0,22 & $-3,4 \%$ \\
4,5 & 0,56 & $-0,6 \%$ & 0,30 & $-2,3 \%$ & 0,18 & $-2,5 \%$ \\
5 & 0,40 & $-5,1 \%$ & 0,23 & $-2,8 \%$ & 0,14 & $-3,4 \%$ \\
\hline
\end{tabular}

\section{Relative Abweichung der gemessenen Wasserenergiedosis bezogen auf die Dosisangaben} des Intrabeamsystems

Die Messung der Wasserenergiedosis an der Applikatoroberfäche zeigte eine mittlere Abweichung (über alle Applikatoren) von -3,2\%. Die kleinste Abweichung betrug $-0,6 \%$ bei einem Applikatordurchmesser von 4,5 cm. Die größte Abweichung lag bei $-5,3 \%$ für den Applikator mit dem Durchmesser 3,5 cm.

In 5 mm Tiefe RW-1 fanden wir im Mittel eine Abweichung von $-4,6 \%$, wobei die Abweichung der Dosis bei den Applikatoren mit Durchmesser 3,0 cm, 3,5 cm, 4,5 cm und $5,0 \mathrm{~cm}$ zwischen $-2,3 \%$ und $-4,9 \%$ lag. Eine Ausnahme bildete der Applikator mit $4 \mathrm{~cm}$ Durchmesser mit einer Dosisabweichung von $-10,0 \%$.

In $10 \mathrm{~mm}$ Tiefe waren die Ergebnisse vergleichbar, die mittlere Abweichung betrug $-4,3 \%$. Bei dieser Messreihe wich der Applikator mit Durchmesser 3,5 cm mit -8,7\% am weitesten $\mathrm{ab}$. 


\section{Bestimmung der Messunsicherheit}

Um die Reproduzierbarkeit unserer Messwerte zu überprüfen wurde nach einer Messung der Applikator neu positioniert und die Messung wiederholt. Die mittlere Abweichung zwischen den Messwerten lag bei 0,38\% mit einer Standardabweichung (SD) von 1,06\%. Für die Bestimmung der Messunsicherheit wurde von folgenden Werten ausgegangen: Positionierung der Kammer $\pm 2,0 \%$, Kalibrierung der Kammer Kalibrierschein PTW $\pm 4,0 \%$, Anzeige Unidos Kalibrierschein PTW $\pm 0,5 \%$, Einfluss abweichender Strahlenqualität $\pm 2,0 \%$, Verwendung von RW $1 \pm 2,0 \%$, sonstige Einflüsse (Unsicherheiten bei Luftdichte, Temperatur) $\pm 2,0 \%$. Die Unsicherheitsanalyse für unseren Messaufbau ergab $\pm 6,0 \%$. Die Messunsicherheit für die Bestimmung der Tiefendosiskurve der geräteinternen Dosimetrie wurde vom Hersteller mit $6 \%$ angegeben.

\section{Konstanzprüfung: Symmetrie der Röntgenstrahlung; Dosisleistung}

Die mit dem PDA für die Raumrichtungen $\mathrm{x}, \mathrm{y},-\mathrm{x},-\mathrm{y}$ gemessenen Werte sind in der Abbildung 2 und und für $\mathrm{z}$ in Abbildung 3 graphisch dargestellt. Die mittlere Abweichung bezüglich des Messwerts in z-Richtung betrug für $\mathrm{x}-2,7 \%$ (SD 1,23\%), y-4,1\% (SD 0,79), $-\mathrm{x}-7,1 \%$ (SD 0,81$)$, und für $-\mathrm{y}-5,3 \%$ (SD 1,01).

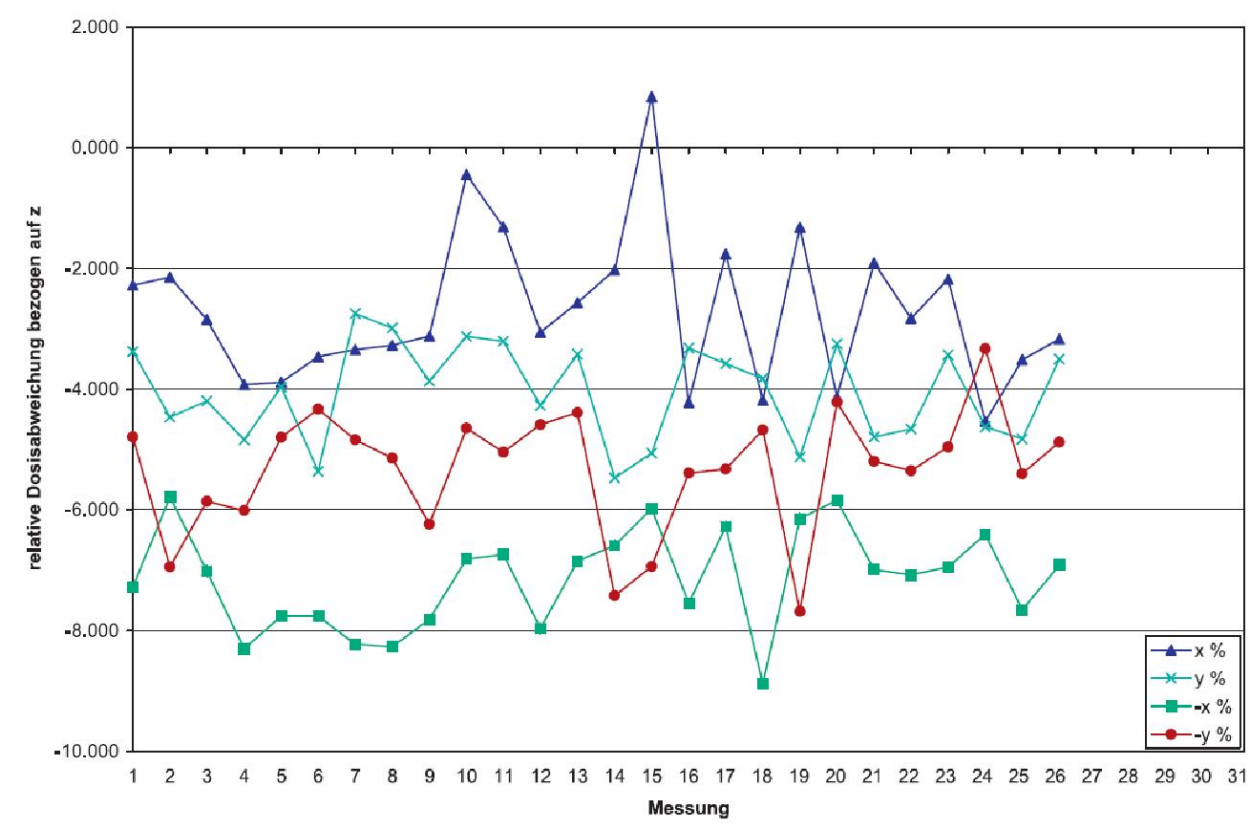

Abbildung 2.

Auswertung der Konstanzprüfung bezüglich der Symmetrie in der Ebene x, y senkrecht zur Geräteachse z. Angegeben ist die relative Abweichung bezogen auf $\mathrm{z}$. 


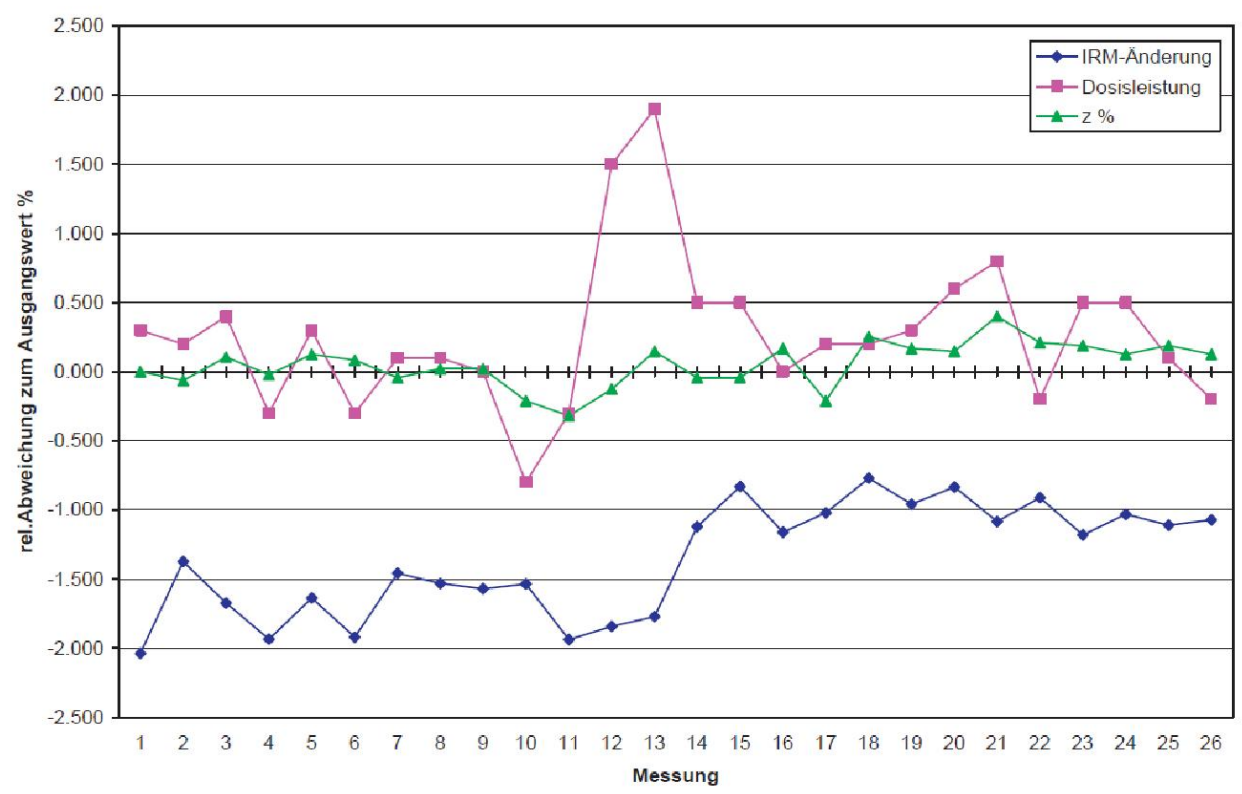

Abbildung 3.

Vergleich der Ergebnisse der arbeitstäglichen Konstanzprüfung. Aufgetragen sind die relativen Abweichungen zum Ausgangswert (Kalibrierfile Hersteller, Diode erste Messung); interner Strahlenmonitor (IRM) blau, am Referenzort gemessenen Dosisleistung magenta, Diodenmesswert in z-Richtung (Geräteachse) grün.

Die relativen Abweichungen der Messwerte des internen Strahlenmonitors IRM, der Diode (PDA) in z-Richtung sowie der Dosisleistungsmessung zum Ausgangswert im Referenzpunkt (PAICH) sind in Abbildung 3 dargestellt.

Die mittleren Abweichungen bezogen auf die Ausgangswerte betrugen für die IRM Messwerte $-1,4 \%$ (SD 0,39), für die Diode (PDA) in z-Richtung $<0,1 \%$ (SD 0,16) und für die Messung der Dosisleistung im Referenzpunkt 0,3\% (SD 1,01)

\section{Diskussion}

Für dosimetrische Vergleiche zwischen verschiedenen Einrichtungen beziehungsweise Bestrahlungssystemen reichen standardisierte Vorrichtungen [16] aus. Hier findet keine Überprüfung der Wasserenergiedosis des Gesamtsystems Strahlenquelle - Applikator statt. Aufwändigere Messungen beziehungsweise Berechnungen mit analytischen oder MonteCarlo-Modellen [10] wurden bereits vorgestellt. Eine gute Möglichkeit zur Dosimetrie bilden auch GafChromic Filme. Die Filme sollten für einen Energiebereich von $20 \mathrm{kV}$ bis $50 \mathrm{kV}$ Röntgenstrahlung geeignet sein. Die Wahl des Scanners kann die Ergebnisse der Dosimetrie beeinflussen, er ist daher auf seine Eignung zu prüfen. Für die Herstellung eines geeigneten Phantoms sollte Material verwendet werden, welches bei dem verwendeten Energiespektrum dosimetrisch wasseräquivalent ist [17] and [18]. Ein Ziel dieser Arbeit war, eine einfache Methode zur Überprüfung der Wasserenergiedosis vorzustellen, die auch unter schwierigen Vorausetzungen umsetzbar ist und die keiner größeren zusätzlichen Anschaffungen bedarf. Schwierigkeiten bereitete hierbei der steile Gradient der Tiefendosis. Probleme durch Unsicherheiten bezogen auf die Messtiefe konnten durch das Aufsetzen des Applikators auf die Phantomoberfläche vermieden werden. Die meisten Ergebnisse stimmen in einem Bereich zwischen $-1 \%$ und $-5 \%$ mit den Messungen des Herstellers überein. Lediglich zwei Messungen lagen außerhalb dieses Bereiches. Berücksichtigt man die angegebenen Messunsicherheiten, so liegen auch diese beiden Werte innerhalb dieser Grenzen. 
Ein weiteres Ziel der Arbeit war die Untersuchung der Langzeitstabilität des Gerätes. Bei Einzeitbestrahlungen ist es von klinischer Bedeutung, dass sich die Abweichung der verabreichten Dosis innerhalb der Toleranzgrenzen befindet. Dieses wird durch den arbeitstäglichen Check, der eine Konstanzprüfung der Dosisleistung beinhaltet, als auch den internen Strahlenmonitor IRM, welcher die Dosisleistung während der Behandlung überwacht, erreicht. Die Messergebnisse belegen sowohl eine hohe Stabilität der Dosisleistung, als auch des internen Strahlenmonitors IRM. Lediglich ein leichter Anstieg der Dosisleistung und damit auch des IRM nach der Wartung (Abb. 3 Messung 14) war zu beobachten.

Für die Beurteilung der Symmetrie ist zu beachten, dass die Werte der Dioden $\pm \mathrm{x}$ und $\pm \mathrm{y}$ relativ zu z angegeben werden. Das Gerät sieht diesen Check als nicht bestanden an, wenn die relative Abweichung mehr als $12 \%$ beträgt. Dies könnte bedeuten, dass der Test mit einem Wert für Diode $\mathrm{x}$ von $11 \%$ und einem Wert von $-11 \%$ für Diode $-\mathrm{x}$ als bestanden gewertet wird. Daher ist hier auch auf den relativen Unterschied der Dioden untereinander zu achten. In Abbildung 2 können Abweichungen bis maximal 7\% beobachtet werden.

\section{Zusammenfassung}

Unsere Überprüfung der Wasserenergiedosis bestätigte die Dosimetrie des Herstellers. Insgesamt erwies sich das System Intrabeam innerhalb der Hersteller-Spezifikationen als stabil.

\section{Literatur}

[1] J.-S. Vaidya, M. Baum, J.-S. Tobias, S. Morgan, D. D'Souza: The novel Technique of delivering targeted intraoperative radiotherapy (Targit) for early breast cancer. Eur J Surg Oncol, 28 (2002), pp. $447-454$

[2] R. Sauer, M.-L. Sautter-Bihl, W. Budach, et al.: Accelerated partial breast irradiation: consensus statement of 3 German Oncology societies. Cancer, 110 (2007), pp. 1187-1194

[3]_U. Kraus-Tiefenbacher, L. Bauer, A. Scheda, et al.:Intraoperative radiotherapy (IORT) is an option for patients with localized breast recurrences after previous external-beam radiotherapy. BMCCancer, 7 (2007), p. 178

[4] O. Nairz, H. Deutschmann, M. Kopp, et al. A dosimetric comparison of IORT techniques in limited-stage breast cancer. Strahlenther Onkol, 182 (2006), pp. 342-348

[5] U. Kraus-Tiefenbacher, A. Scheda, V. Steil: Intraoperative Radiotherapy (IORT) for breast cancer using the IntraBeam ${ }^{\mathrm{TM}}$ system. Tumori, 91 (2005), pp. 339-345

[6] C. Herskind, V. Steil, U. Kraus-Tiefenbacher, et al.: Radiobiological Aspects of Intraoperative Radiotherapy (IORT) with Isotropic Low-Energy X-rays for Early-Stage Breast Cancer. Radiat Res, 163 (2005), pp. 208-215

[7] Richtlinie zur Durchführung der Qualitätssicherung bei Röntgeneinrichtungen zur Untersuchung oder Behandlung von Menschen nach den $\S \S 16$ und 17 der Röntgenverordnung-QualitätssicherungsRichtlinie (QS-RL). Gemeinsamen Ministerialblatt 2003;783. 
[8] Bekanntmachung einer Empfehlung der Strahlenschutzkommission ,Strahlenschutz in der Röntgentherapie" Vom 18. Mai 2006 (BAnz. Nr. 108 vom 10.06.2006 S. 4325) 〈http://www.umweltonline.de/regelwerk/energie/strahlen/roen_ges.htm〉 .

[9] Peinsippl N, Roos G, Weimer G. Röntgenverordnung RöV Heidelberg Forkel Verlag; 2006.

[10] M.-A. Ebert, B. Carutthers: Dosimetric characteristics of a low-kV intra-operative X-ray source: Implications for use in a clinical trial for treatment of low-risk breast cancer. Med Phys, 30 (2003), pp. 2424-2431

[11] Armoogum K-S, Parry J-M, Souliman S-K, et al. Functional intercomparison of intrapoperative radiotherapy equipment-photon radiosurgery system. Radiat Oncol 2007;2: doi:10.1186/1748-717X-211

[12] Schweizerische Gesellschaft für Strahlenbiologie und Medizinische Physik. Dosimetrie von Röntgenstrahlen im niederen und mittleren Energiebereich. Empfehlungen 2001;9.

[13] DIN 6809 Teil4. Berlin: Beuth Verlag; 1988.

[14] Heuß K, Bunde E, Dierker J, et al. Praxis der Weichstrahldosimetrie. DGMP-Bericht 1986;5.

[15] INTRABEAM ${ }^{\circledR}$ System PRS 500 mit XRS 4Software-Version 2.1 Gebrauchsanweisung für das Kernsystem. 2007 G-30-1601-de.

[16] K. Armoogum, C. Watson: A dosimetry intercomparison phantom for intraoperative radiotherapy. Z Med Phys, 18 (2008), pp. 120-127

[17] Schiebel R. Evaluation der 2-D Bestrahlungsplanung durch Task Group No. 43 Protokoll mit GafChromik Filmdosimeter Diplomarbeit im Studiengang Medizintechnik Fachhochschule GiessenFriedberg; 2005.

[18] Schneider F. Entwicklung eines Verfahrens zur intracavitären Bestrahlung mit einem intraoperativen Bestrahlungsgerät Fachhochschule Wiesbaden; 2005.

Copyright (C) 2009 Published by Elsevier B.V. 